\section{VISFATIN INCREASES INVASION IN HUMAN ADULT GRANULOSA TUMOR CELL LINE BY STIMULATION OF GLUCOSE METABOLISM REPROGRAMMING}

J Gogola-Mruk*, K Krawczyk, W Marynowicz, A Ptak. Jagiellonian University, Institute of Zoology and Biomedical Research, Laboratory of Physiology and Toxicology of Reproduction, Kraków, Poland

\subsection{6/ijgc-2021-ESG0.357}

Introduction/Background* Adult granulosa cell tumors (AGCT) accounting about 95\% GCT (granulosa cell tumors) is characterized by the occurrence of an aggressive form and frequent appearance of recurrence and metastasis. ${ }^{1}$ Furthermore, reports indicated that the level of visfatin, which is one of adipokine, is elevated in ascites fluid of ovarian cancer patients with diagnosed metastases. ${ }^{2}$ Moreover, the role of visfatin in cancer metabolic reprogramming was demonstrated. ${ }^{3}$ Therefore, the aim of this research is to determine whether visfatin by reprogramming metabolism may be involved in invasion of AGCT

Methodology Human AGCT-derived cell line KGN (Riken Cell Bank) was cultured in three-dimensional culture. Spheroids were exposed to visfatin $(100 \mathrm{ng} / \mathrm{ml})$ for $24 \mathrm{~h}$ and invasion was measured using Culturex Spheroid Invasion Matrix (\#3500-096-03; Trevigen). Glucose uptake was tested using a Glucose Uptake-Glo Assay (Promega) after treatment with visfatin $(100 \mathrm{ng} / \mathrm{ml})$ for $24 \mathrm{~h}$. Silencing of NAMPT (visfatin gene) was carried out using siRNA (SMART pool siRNA NAMPT; L-004581-00-0005) and basal glycolysis was measured using Seahorse Glycolytic Rate Assay (Agilent).

Result(s)* Firstly, we showed that visfatin increases KGN cells spheroids invasion. Furthermore, we observed stimulating glucose uptake in KGN cells after treatment with visfatin. Whereas the use of STF-31 (a blocker for the GLUT1 and an inhibitor of visfatin) abolished the stimulating effect of visfatin on the invasion, which indicates the important role of glucose metabolism in this process. Moreover, silencing of NAMPT decreased basal glycolysis in KGN cells spheroids.

Conclusion* These results stated that visfatin by reprogramming glucose metabolism plays an important role in increasing the ability of AGCT to invasion and because of that it can be a factor to progression of this cancer.

This study was funded by the National Science Centre (NCN) Poland (grant number 2019/33/N/NZ5/00471)

\section{REFERENCES}

1. Sonoyama A, Kanda M, Ojima Y, et al. J Med Sci 2015:61:E109-14.

2. Li Y, Li X, Liu KR, et al. Eur J Cancer Prev 2015:24;231-9.

3. Audrito V, Manago A, Gaudino F, et al. Semin Cell Dev Biol 2020;98:192-201.

\section{THE IMPACT OF CHEMOTHERAPY RESPONSE SCORE AND LYMPHOCYTIC INFILTRATION IN PATIENTS WITH OVARIAN CANCER TREATED WITH NEOADJUVANT CHEMOTHERAPY}

\footnotetext{
${ }^{1}$ I Rodolakis*, ${ }^{1} \mathrm{~A}$ Prodromidou, ${ }^{2} \mathrm{M}$ Sotiropoulou, ${ }^{3} \mathrm{M}$ Liontos, ${ }^{1} \mathrm{D}$ Haidopoulos, ${ }^{3} \mathrm{~A}$ Bamias, ${ }^{1} \mathrm{D}$ Loutradis, ${ }^{1} \mathrm{~N}$ Thomakos, ${ }^{1} \mathrm{~A}$ Rodolakis. ${ }^{1} 1 \mathrm{st}$ Department of Obstetrics and Gynecology, Medical School, National and Kapodistrian University of Athens, 'Alexandra' Hospital, Athens, Greece; 'Department of Pathology, General Hospital "Alexandra", Athens, Greece, ${ }^{3}$ Department of Clinical Therapeutics, School of Medicine, National and Kapodistrian University of Athens, Alexandra Hospital, Athens, Greece
}

10.1136/ijgc-2021-ESG0.358
Introduction/Background* Neoadjuvant chemotherapy (NACT) followed by interval debulking surgery (IDS) represents an alternative approach to primary debulking for the management of advanced epithelial ovarian cancer (EOC) with comparable survival outcomes. Histopathologic examination of the IDS specimens and the evaluation of Chemotherapy Response Score (CRS) have been proposed as significant markers of response to NACT. The aim of the prsent study was to evaluate the prognostic significance of CRS in OC patients treated with NACT.

Methodology A single institution retrospective analysis of patients with OC stage $\geq$ III who were selected to receive NACT from 2011 to 2018 was performed. Omental and ovarian samples were assessed for the evaluation of CRS according to International Collaboration on Cancer Reporting (ICCR) recommendation. Lymphocytic infiltration, presence of necrosis and mitotic index were also assessed.

Result(s)* The final analysis included a total of 60 patients with median age of 65 years at diagnosis. Omentum and ovarian samples of the included patients revealed a CRS 3 in 20 and 10 patients, respectively. Patients with CRS3 at omentum had significantly prolonged median progression free survival (PFS) compared to CRS1 and CRS2 (19 vs 10 vs 15 months, respectively, $p=0.002$ ), while no difference was observed in the respective OS among the groups (42 vs 28 vs 32.3 months, respectively). Lymphocytic infiltration in pre-treatment biopsies was related to improved PFS and OS (log-rank p $=0.01$ and $p=0.015$, respectively). No effect on either PFS or OS was observed in patients who received bevacizumab postIDS, while in patients with lymphocytic infiltration bevacizumab negatively affected PFS.

Conclusion* CRS seems to play a key role in the prediction of the postoperative course of EOC patients who received NACT and IDS. However, further studies are warranted to decipher the exact role of CRS and lymphocytic infiltration so as to personalize the treatment of EOC patients.

\section{SKIN CAPILLARY DENSITY AS A NOVEL BIOMARKER OF CYTOTOXIC AND BEVACIZUMAB TREATMENT IN OVARIAN CANCER: A PROSPECTIVE CLINICAL TRIAL}

${ }^{1} \mathrm{G}$ Cass*, ${ }^{2} \mathrm{R}$ Lewes, ${ }^{3} \mathrm{~J}$ Pawade, ${ }^{1} \mathrm{C}$ Newton. 'University Hospitals Bristol NHS Foundation Trust Headquarters, UK; ${ }^{2}$ University of Bristol, UK; ${ }^{3}$ North Bristol NHS Trust, UK

\subsection{6/ijgc-2021-ESG0.359}

Introduction/Background* The role of angiogenesis in ovarian cancer progression is well established. Bevacizumab has been demonstrated to have a survival advantage for a subgroup of women with stage 4 disease and those who have suboptimal debulking surgery. The search for a biomarker of Bevacizumab efficacy is elusive. Skin capillary density (SCD) is a dynamic marker that may provide a surrogate indicator of angiogenic activity and alter in response to treatment in cancer patients. Methodology A cohort of 50 women with stage 3 and 4 high grade serous cancer were enrolled over 2 years at University Hospitals Bristol, UK. Written informed consent was obtained from each participant. SCD was measured at baseline and at specified time points during treatment. Serum angiogenic markers (VEGF and Ang 1) and tissue micro vessel density and proliferation (ki67) were also measured and correlated with SCD. Longitudinal and survival analysis was conducted to ascertain changes in SCD during treatment and association 